\title{
An effective User Interface Image Processing Model for classification of Brain MRI to provide prolific Healthcare
}

\author{
Vinit Kumar Gunjan $^{1}$, M Janga Reddy ${ }^{2}$, Fahimuddin.Shaik ${ }^{3}$, V. Hymavathi ${ }^{4}$ \\ ${ }^{1,2}$ CMR Institute of Technology, Hyderabad, 501401, Andhra Pradesh, India \\ ${ }^{3,4}$ Annamacharya Institute of Technology \& Sciences, Rajampet, 516126, Andhra Pradesh, India \\ Email : vinitkumargunjan@gmail.com, principal@cmritonline.ac.in, fahimuddin.shaik.in@ieee.org, \\ hymavalluru55@gmail.com
}

Received: $5^{\text {th }}$ March 2018, Accepted: $9^{\text {th }}$ April 2018, Published: 30 $^{\text {th }}$ April 2018

\begin{abstract}
Today the design and development of computer assisted User Interface Image processing Systems have been very helpful in detecting and diagnosing the underlying diseases in the early stage. Out of many anomalous diseases, Brain Tumor Diagnosis and treatment has been a challenge to medical as well as to research community. If it is detected at an early stage the chances of saving a life are more. The primary goal of this work is to develop an effective User Interface Image Processing Model via a GUI (Graphical User Interface) for brain tumor detection and segmentation. The methods amalgamated together and investigated here are Fast Bounding Box (FBB) method and Discrete Wavelet Transform (DWT) with Morphology \& SVM (Support Vector Machine) Classifiers.
\end{abstract}

Keywords: Brain Tumor, Detection, filtering, image,

\section{INTRODUCTION}

The uncharacteristic growth of cells in the brain prompts Brain tumor. Normally brain tumor rises up from brain cells, blood vessels or nerves that are existing in the brain. Early recognition of brain tumor is vital as death rate is higher among people having brain tumor [1]. As per 2007 estimates absolutely 80,271 people are affected by different sorts of tumor in India [6].Techniques for brain tumor identification utilizing image processing have been available for couple of decades. Researchers have proposed numerous semi-automatic in addition to automatic image processing techniques for identifying brain tumors however the majority of them fail to give effective and exact outcomes because of the presence of noise, inhomogeneity, poor images contrast that arise typically in medical images [4]. The significance of this proposal is to design and develop a complete automated diagnosis system for brain tumor, including removal of noise, detection of the brain tumor, extraction as well as classification of brain tumor. This paper proposes amalgamation of two methods, Fast Bounding Box method and DWT with Morphology \& SVM Classifiers . Even though some studies suggest that segmentation of brain tumor from MRI Images is time consuming procedure but it is an important research to be carried out [5].

\section{Proposed Work}

The proposed method is represented in below diagram.

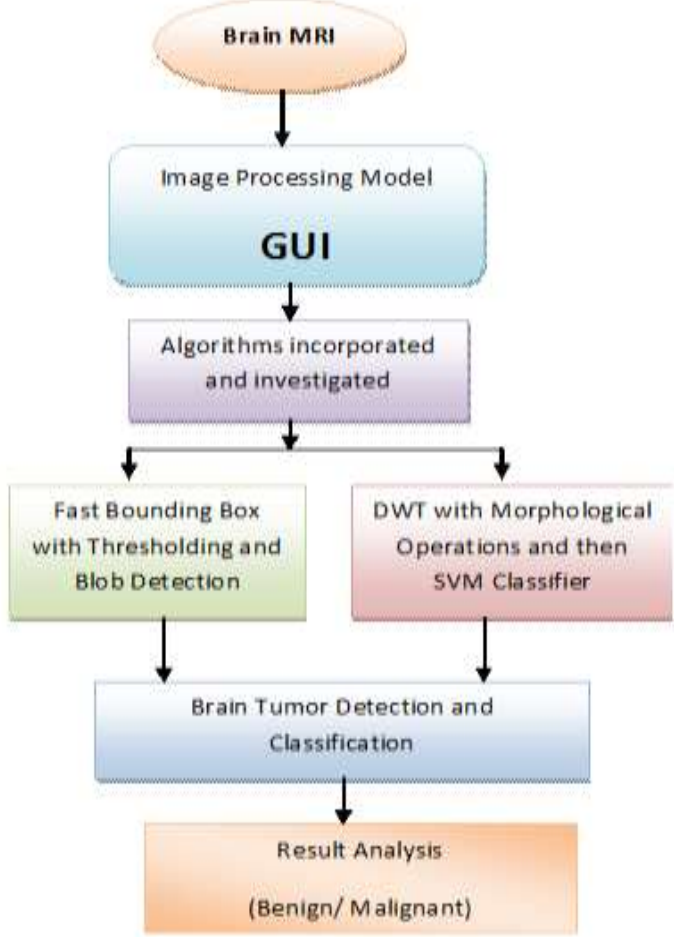

Fig. 1. Block Diagram of Proposed Method

Once the image acquisition is completed, it is fed to algorithms incorporated in the GUI. The First method to be investigated is FBB and here the initial step augments the image and then fast bounding box algorithm is used for detecting the region of interest in second step and finally post processing operations are utilized for segmenting the brain tumor in third step. As the consequence of above steps, a final brain tumor segmented image will be obtained. The second method to be investigated is chiefly concerned with Classification of Brain Tumor via DWT with Morphology \& SVM Classifiers, whether it is benign or malignant.

\section{A. Implementation Procedure}

For this work, the input images acquired from different open databases were simulated with the help of Matlab (Matrix Laboratory), a high-level Technical Computing language (R2010a version and above) on an Intel Core i5 processor PC which runs at $2.5 \mathrm{GHZ}$ having a total physical memory of $4 \mathrm{~GB}$ 
RAM. More than 100 toolboxes accessible in Matlab, yet just a couple of, for example, image acquisition, image processing, fixed point and neural networks tool boxes have been utilized and it also contain a number of block sets, which contain a set of instructions belong to same category or family of execution. Matlab is a trademark product of Mathworks Inc. which permits matrix manipulations [3], image is considered as a matrix for operation and output obtained is also stored in form of a matrix.

\section{MethodologY}

This research work mainly involves with two methods as said in previous sections and they are
A. FBB with Thresholding and Blob Detection
B. DWT with Morphology \& SVM Classifiers

B. FBB with Thresholding and Blob Detection

The three processes of significance in this method are Preprocessing, Region of Interest and Post Processing which are explained in an obvious manner. These methods involves thresholding and blob detection and is represented in below diagram

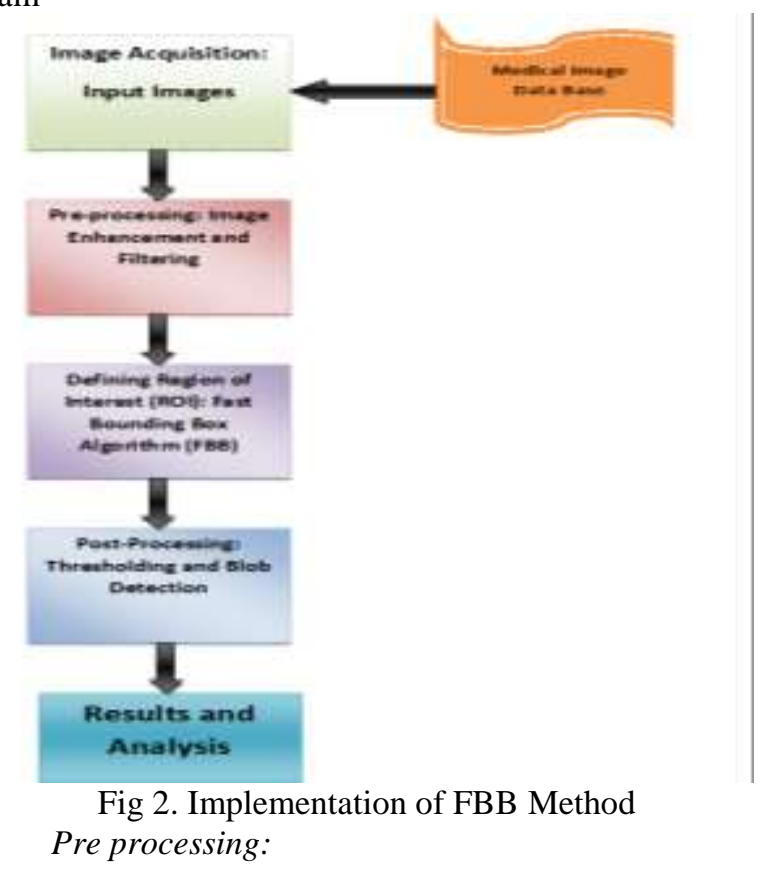

Preprocessing is the initial phase in the proposed technique. Noise involved while acquisition of images through medical imaging modality is removed and enhances the image for further processing.

These steps enhance quality of the image in addition to that it also increases accuracy and surety in detection of tumor. preprocessing stage consists of the following steps as mentioned:-

1) conversion of image into grayscale.

$$
\mathrm{f}^{\wedge}(\mathrm{x}, \mathrm{y})=\operatorname{median}(\mathrm{s}, \mathrm{t})\{\mathrm{g}(\mathrm{s}, \mathrm{t})\} \text {. }
$$

2) Application of median filter of about $3 \times 3$ on the image using equation 1 keeping in mind the end goal to remove noise [8].

3) The acquired image is then passed through a high pass edge detection filter ( Anisotropic diffusion is used to remove noise as this method involves in removing the unwanted noise and artifacts acquired during image acquisition without losing the integrity of the image i.e. lines and edges of the image are preserved for future work)

4) The noticed edges are added to the actual image for enhancing its edges. This helps in easy detection as well as segmentation of the tumor.

ii. Region of Interest

Fast Bounding Box algorithm is utilized in the proposed method for detecting the region of interest (ROI). In general ROI is the tumor area of the image. An unsupervised method which is a change detection, Bounding Box is used here search for the most dissimilar region in subjected MRI image [9].

\section{iii. Post processing:}

After detecting the ROI, we perform few post processing operations for locating the tumor part clearly in the brain. The main reason of doing post processing is to display only that portion of the image $\mathrm{D}$, which is having the tumor. Post processing operations incorporate a few mathematical operations as well as some windowing techniques [7].

C. DWT with Morphology \& SVM Classifiers

The processes involved here are given below with a brief description and they are

i. Gray Thresholding and then application of Morphological opening area operation

ii. Then Single level 2D Wavelet Transform is employed for the decomposition of Image levels.

iii. Using the Loaded available data SVM Classifier is used to classify the type of Tumor, whether it is Benign or Malignant.

\section{Results and Discussion}

A GUI is developed and is presented below which is designed and developed to classify the Brain Tumors from MRI

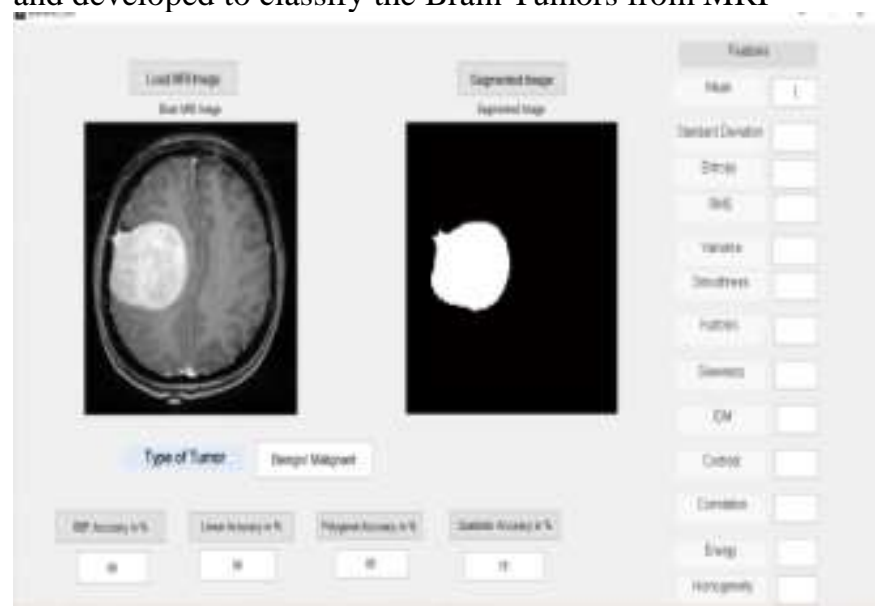

Fig 3. User Interface Image Processing Model 
A coronal fat-suppressed T1-weighted magnetic resonance image has been considered for simulation. The coronal image considered is gained after gadolinium administration which is appeared in Figure 4.

As image is subjected to the developed algorithm, it goes under various procedures as said above in methodology. Original Image is first filtered to remove noise and fed to anisotropic diffusion. The images acquired may contain some noise elements, hence filtering is required. Then the image is subjected to Bound box procedure, later SVM classifier is applied to obtain segmented tumour.

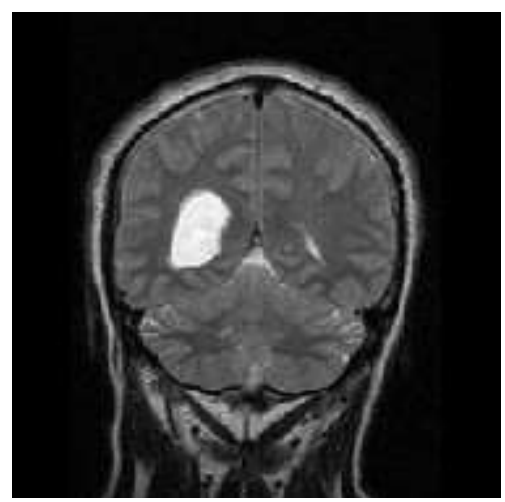

Fig. 4. Original Image

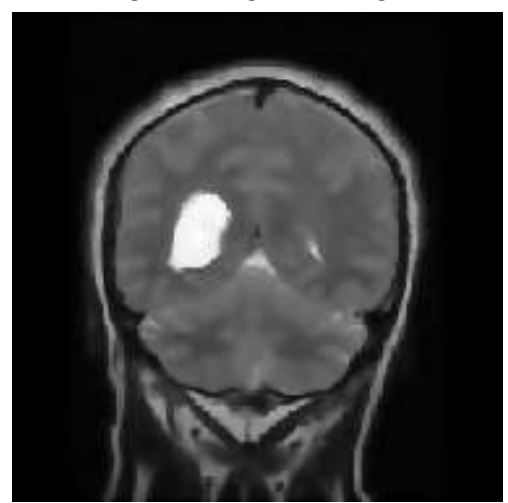

Fig. 5. Filtered Image

Figure 5 is the filtered image which is obtained by using anisotropic diffusion (a 2D Gaussian filter is convolved with the original image which is innate here), an image processing method which aims in removing noise without distorting the edges and lines of the image.

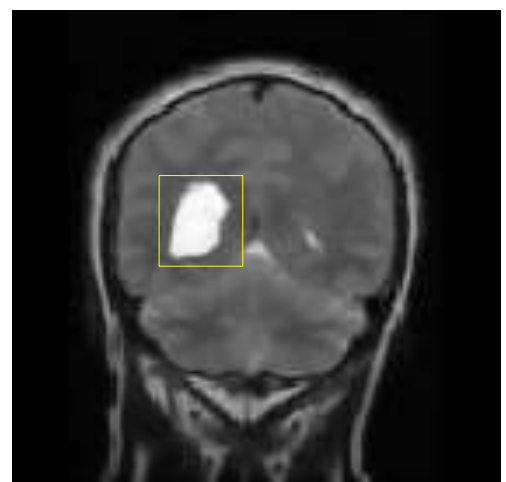

Fig. 6. Locating Bound Box

Figure 6 is the image obtained on applying proposed fast bounding box method which helps in locating the ROI i.e. tumour part of MRI image. It draws itself a rectangle box, which finally aims to locate ROI depending upon the intensity values.

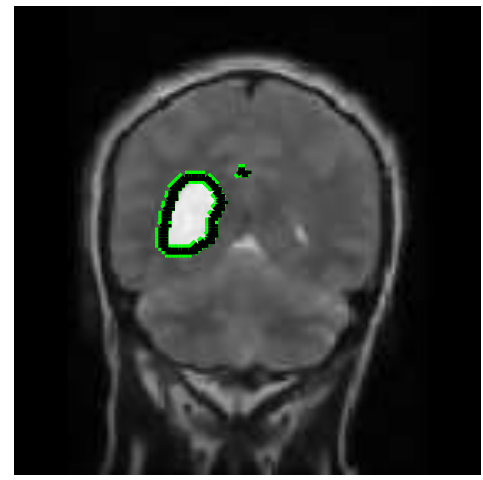

Fig. 7.SVM Classifier resulted image

Figure 7 is the image obtained from SVM Classification, which clearly indicates the region of Brain tumour shown with a borderline representation. This depiction is especially useful to understand the part of the brain affected and may fed to edge detection process to segment out only the tumour part.

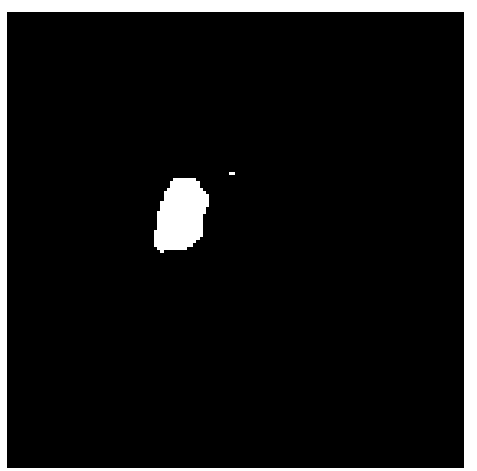

Fig. 8. Tumor Detection

Figure 8 is the final segmented image which clearly indicates the region of Brain tumour separated from original image.

Once this process of Detection of Tumor is completed, the image is classified based on database using SVM Classifier. 


\section{CONCLUSION}

In this work, a coronal fat-suppressed T1-weighted magnetic resonance image is considered for simulation, the coronal image which is considered for this work is obtained after gadolinium administration. The outcomes got have been analyzed with respect to the ROI (damaged part of the brain) which has been observed. This research work is primarily useful as an automatic CAD system for brain Tumor segmentation. As a future scope the images resulted can be verified for statistical approaches to find image attributes which will be helpful in parametric analysis.

\section{ACKNOWLEDGMENT}

The authors are thankful to Annamacharya Institute of Technology \& Sciences, Rajampet, A.P. for providing research facilities.

\section{REFERENCES}

[1] Akram, M. Usman, and Anam Usman. "Computer aided system for brain tumor detection and segmentation", International Conference on Computer Networks and Information Technology, 2011.

[2] Saha, B.N.. "Quick detection of brain tumors and edemas: A bounding box method using symmetry", Comput Med Imaging Graph. 2012 Mar;36(2):95-107

[3] http://www.mathworks.se/products/matlab/

[4] Chaudhari, A. K., and J. V. Kulkarni. "Local entropy based brain MR image segmentation", 2013 3rd IEEE International Advance Computing Conference (IACC), 2013.

[5] Fahimuddin. Shaik, Dr.Anil Kumar Sharma, Dr.Syed. Musthak Ahmed, "Hybrid Model for Analysis of Abnormalities in Diabetic Cardiomyopathy and Diabetic Retinopathy related images", Springer Plus Journal, Springer Publications, ISSN:2193-1801

[6] Farmer, M.E, Jain, A.K., "A wrapper-based approach to image segmentation and classification”, Pp: 2060 - 2072, Image Processing, IEEE Transactions on journals and magazines, Dec. 2005.

[7] Vinit Kumar Gunjan, Fahimuddin.Shaik, C.Venkatesh, M.Amar Nath, "Computational Methods In Molecular Imaging Technologies", Springer-Nature Publishers(Springer Briefs in Applied Sciences and Technology-Forensics and Medical Bio-informatics), Singapore, June 2017.( ISBN 978-981-10-4635-3)

[8] T. Logeswari and M. Karnan, An improved implementation of brain tumor detection using segmentation based on soft computing, Second International Conference on Communication Software and Networks, 2010. ICCSN ${ }^{\text {ee } 10 . P a g e(s): ~ 147-151 . ~}$

[9] Fahimuddin. Shaik, Dr.Anil Kumar Sharma, Dr.Syed. Musthak Ahmed, Vinit Kumar Gunjan and Chandra Naik "An improved model for analysis of Diabetic Retinopathy related imagery", Indian Journal of Science and Technology, 2016 ISSN: 0974-6846 